\title{
Derivation of Regge Trajectories from the Conservation of Angular Momentum in Hyperbolic Space
}

\author{
B.H. Lavenda* \\ Università degli Studi, Camerino 62032, MC, Italy
}

\begin{abstract}
Regge trajectories can be simply derived from the conservation of angular momentum in hyperbolic space. The condition to be fulfilled is that the hyperbolic measure of distance and the azimuthal angle of rotation in the plane be given by the Bolyai-Lobachevsky angle of parallelism. Resonances or bound states that lie along a Regge trajectory are relativistic rotational states of the particle that are quantized by the condition that the difference in angular momentum between the rest mass, assumed to be the lead particle on the trajectory, and the relativistic rotational state is an integer. Since the hyperbolic expression for angular momentum is entirely classical, it cannot take into account signature, and whether exchange degeneracy is broken or not. However, the changes in the angular momentum are in excellent agreement with non-exchange degeneracy which assumes four different rest masses in the case of $\rho, \omega, \mathrm{a}_{2}$, and $\mathrm{f}$ mesons trajectories, as well as in the case where there is only one rest mass in exchange degeneracy. A comparison is made between the critical and supercritical Pomeron trajectories; whereas the former has resonaces with higher masses, it has corresponding smaller linear dimensions.
\end{abstract}

Keywords: Regge trajectories, conservation of angular momentum in hyperbolic space, relativistic rotational states.

\section{INTRODUCTION}

Regge trajectories link bound states and resonances with the same internal quantum numbers but with different values of the mass, $\mathrm{m}$, and angular momentum, $J$. When the $J$ values of the meson, or baryon, groups are plotted against the square of the mass linear trajectories result. They are known as Chew-Frautschi plots [1]. Regge theory cannot account for the linearity of these trajectories, and string theories cannot account for the intercept of these trajectories [2].

It also became clear that many of the trajectories with different quantum numbers coincide. This became known as the principle of exchange-degeneracy [3]. The weak form of this principle states that only the trajectories with different quantum numbers coincide, while the strong form, which has subsequently been refuted by experiment, says that in addition the corresponding residue of the hadronic amplitude coincides with a given pole in the complex angular momentum plane. Weak exchange degeneracy was believed to be due to the opposite signature of otherwise identical trajectories [4], and seemed to violate the principle that a Regge trajectory produces a physical bound state, or resonance, each time $\Delta J$ increases by a factor of two, and not one [5].

The string model considers quarks and antiquarks for mesons to be held together by the color field. Unlike the lines of force of electric charges which tend to spread out, the gluon-gluon interaction pulls the lines together in the form of a tube which rotates about the axis through the

*Address correspondence to this author at the Università degli Studi, Camerino 62032, MC, Italy; Tel.+390737402216;

E-mail: info@bernardhlavenda.com center of mass of the tube. The motion is considered relativistic, but since a uniform rotating string is not an inertial system, the Lorentz contraction cannot be employed. The total mass is expressed in terms of the angular momentum and the invariant pressure of the bag which exactly balances the vacuum pressure. To find the optimum length of the tube, the total mass is minimized with respect to the length at constant angular momentum. The stationary condition sets the angular momentum proportional to the square root of the invariant pressure. Asymptotically a linear trajectory is obtained, but the angular momentum is then no longer a constant, independent of the length of the tube, and consequently of the total mass, as it was assumed in the variational procedure to obtain the optimum length of the tube.

It is the purpose of this paper to show that Regge trajectories are relativistic rotational states of the particle trajectory that are quantized by their $\Delta J$ values. Since there is no integration over the length of a tube, the intercept is obtained in terms of the mass and frequency of rotation of the particle whose resonances along the same linear trajectory are relativistic rotational states of the particle. In order to carry out the program, we must digress to discuss relativistic motion of a uniform rotating disc or string.

\section{HYPERBOLIC MOTION OF A UNIFORMLY ROTATING STRING}

It was Riemann's original idea that to free himself of the geometries of surfaces in $\mathbf{E}^{3}$, it was necessary to modify the definition of the dot product, $\cdot$, on tangent vectors, and to replace it by some more general inner product that would be applicable to any abstract surface not in $\mathbf{E}^{3}$. The hyperbolic inner product, ${ }^{\circ}$, which involves a sign change in the denominator of the stereographic inner product, is [6]: 
$\vec{x} \circ \vec{y}=\frac{\vec{x} \bullet \vec{y}}{1-\left(x^{2}+y^{2}\right) /(2 R)^{2}}$,

where $K=-1 / R^{2}$ is the constant, negative, Guassian curvature. As the radius of curvature, $R \rightarrow \infty$, we recover the dot product of Euclidean geometry. The stereographic inner product is obtained by allowing the radius, $R$ to become imaginary, $R \rightarrow$ $i R$, thereby changing the sign in the denominator of (1).

The inner product, (1), means that we have to modify the Euclidean metric in the plane to become [7]:

$d s=\frac{\sqrt{d r^{2}+r^{2} d \varphi^{2}}}{1-r^{2} \omega^{2} / c^{2}}$,

which is expressed in polar coordinates $(r, \varphi)$. The metric (2), describing a uniformly rotating disc of velocity $v=\omega r$, is hyperbolic with an absolute constant whose numerical value will depend on the arbitrary choice of a unit segment, $2 R=c / \omega$, the ratio of the speed of light, $c$, to the angular velocity, $\omega$.

Since $\varphi$ is a cyclic coordinate, we know that a first integral exists:

$\frac{r^{2} \varphi^{\prime}}{\left(1-r^{2} \omega^{2} / c^{2}\right) \sqrt{1+r^{2} \varphi^{\prime 2}}}=\frac{\Delta J}{m_{0} c}=$ const.

which is obtained from the stationary condition of the integral of (2) with respect to $\varphi^{\prime}$. All we know, so far, is that the constant of integration has dimensions of length. Solving (3) for $\varphi^{\prime}$, we get

$\varphi^{\prime}= \pm \frac{\left(\Delta J / m_{0}\right)\left(1-r^{2} \omega^{2} / c^{2}\right)}{r^{2} \sqrt{\left.1-\Delta J / m_{0} c r\right)^{2}\left(1-r^{2} \omega^{2} / c^{2}\right)^{2}}}$.

If we set

$\Delta J\left(1-r^{2} \omega^{2} / c^{2}\right)=m_{0} r^{2} \omega$,

showing that $\Delta J$ has units of angular momentum, if $\mathrm{m}_{0}$ is the mass, then (4) will reduce to

$\varphi^{\prime}= \pm \frac{\omega}{c \sqrt{1-r^{2} \omega^{2} / c^{2}}}$

If we choose the negative sign in (6), for $0<\cos ^{-1}(r \omega / c)$ $<\pi$, then upon integrating we will get the equation of a circle,

$r=2 R \cos \varphi$,

with radius $R=c / 2 \omega$.

Rather, if we express $r$ in terms of a segment of a Lobachevsky straight line,

$r=2 R \tanh (\bar{r} / 2 R)$,

we can write (6) as

$\frac{d \varphi}{d \bar{r}}=-(2 r \cosh (\bar{r} / 2 R))$

again choosing the negative sign. Equation (9) can be immediately integrated to give:

$2 \tan ^{-1} e^{-\bar{r} / 2 R}=\varphi$,

which is none other than the fundamental formula of BolyaiLobachevsky for the radian measure of the angle of parallelism. The angle of parallelism must be acute, $\varphi \leq \pi / 2$; this is the reason for have selected the negative sign in (9). Its Euclidean counterpart is a circle of radius $R$, (7). The square of the constant $2 R$ occurs in the proportionality factor of area to defect in hyperbolic geometry.

Rearranging (5) to read:

$\Delta J=J-J_{0}=\frac{m_{0} r^{2} \omega}{1-r^{2} \omega^{2} / c^{2}}=\frac{m_{0} c^{2}}{\omega} \sinh ^{2}(\bar{r} \omega / c)$

we are immediately confronted by the apparent fact that the change in angular momentum, like the angular momentum itself, is not conserved. However, we must realize that if we modify the dot product to the inner product, (1), we must also modify the cross-product,

$\vec{x} \times \vec{y}=\frac{\vec{x} \times \vec{y}}{1-\left(x^{2}+y^{2}\right) /(2 R)^{2}}$,

so that the trigonometric relation, $\cos ^{2} \vartheta+\sin ^{2} \vartheta=1$, is satisfied. Consequently, (11) is an expression of angular momentum conservation in hyperbolic space.

An identical expression for the angular momentum has been obtained in Einstein's general relativity [8]. However, there, it is concluded that (11) "cannot in general be interpreted as angular momentum, since the notion of a 'radius vector' occurring in the definition of the angular momentum has an unambiguous meaning only in a Euclidean space." In contrast to general relativity the 'radius vector', $\bar{r}$, in (8), is defined by the logarithm of the cross-ratio,

$\bar{r}=2 R \tanh -1(r / 2 R)=R \operatorname{In}\left(\frac{1+r / 2 R}{1+r / 2 R}\right)$,

which has an unambiguous meaning eventhough it is not in Euclidean space.

\section{REGGE TRAJECTORIES}

Eliminating the square of the hyperbolic sine between (11), and the square of the relativistic mass,

$$
m^{2}=m_{0}^{2} \cosh ^{2}(\bar{r} \omega / c)=m_{0}^{2}\left\{\sinh ^{2}(\bar{r} \omega / c+1\}\right.
$$

expresses the change in angular momentum to the square of the relativistic mass. We thus obtain the Regge trajectories,

$J=\alpha^{\prime} m^{2}+\alpha(0)$,

with the slope,

$\alpha^{\prime}=\frac{\Lambda}{m_{0} \omega}$,

and intercept,

$\alpha(0)=J_{0}-\frac{m_{0}}{\omega}$,

in natural units, $\hbar=c=1$. Expression (14) can also be written as

$\frac{m_{0}^{2}}{m^{2}}=1-(r \omega)^{2}$,

also in natural units.

It has been claimed [9] that if QCD is the correct theory there should be only a single parameter, either the Regge slope, the scale parameter, or the distance at which the effective charge 
is unity. This length, together with the quark masses, should be sufficient to derive all of hadronic physics. Expression (16) relates the Regge slope to the scale parameter. To maintain units, $\Lambda$ will be taken to be dimensionless, entering as a correction to the angular velocity of rotation. The scale parameter has been determined as $\Lambda=0.16 \pm 0.08 \mathrm{GeV}[9, \mathrm{p}$. 334], where the large relative error is due to the logarithmic relation of scale parameter to the running coupling constant. We will assume $\Lambda=0.2$, which is the numerical conversion factor of $\mathrm{GeV}^{-1}$ to $\mathrm{fm}$, i.e., $1 \mathrm{fm}=5.07 \mathrm{GeV}^{-1}$. $J_{0}$ is the angular momentum of the lead particle of the Regge trajectory whose higher order resonances are relativistic expressions for the angular momenta. They are quantized by the condition that $\Delta J$ is a positive integer. The relativistic nature of the trajectories comes solely from (18) when it is introduced into (11). The parameters $\omega^{-1}$ and $0 \leq r \omega<1$ give the linear dimensions of the particle and the degree of the relativistic state, respectively.

The meson trajectories in Table $\mathbf{1}$ contradict signature, or that the angular momentum must increase in units of two, not one, and were taken from ref. [4]. Since relativistic states do not depend on internal quantum numbers, or signature, the smallness in the relative error in angular momenta differences is quite remarkable.

Actually, the $\rho$-trajectory is a superposition of four distinct trajectories. With the more recent data on resonances, the four trajectories are found to be [3]:

$\alpha_{\rho}\left(m^{2}\right)=0.88 m^{2}+0.478$

$\alpha_{\omega}\left(m^{2}\right)=0.293 m^{2}+0.435$

$\alpha_{\alpha_{2}}\left(m^{2}\right)=0.857 m^{2}+0.512$

$\alpha_{f}\left(m^{2}\right)=0.801 m^{2}+0.697$,

and the calculation of the angular momentum differences in Table 2 shows that the relativistic expressions are as close, if not closer, to those calculated in Table 1. These classic expressions cannot account for differences in the internal quantum numbers and signature.

Table 1. Calculation of the Change in Angular Momentum for Meson-Trajectories

\begin{tabular}{|c|c|c|c|c|c|c|c|}
\hline$\alpha_{2}(1310)$ & & & 0.553 & 0.809 & $J=2$ & 1 & 0.997 \\
\hline$K^{*}(890)$-Trajectory & $\alpha^{\prime}=0.9 \mathrm{GeV}^{-2}$ & $\omega^{-1}=0.7899 \mathrm{fm}$ & $r(\mathbf{f m})$ & $r \omega$ & $J_{0}=1$ & $\Delta J$ & $\Delta J($ cal $)$ \\
\hline$\phi(1020)$-Trajectory & $\alpha^{\prime}=0.9 \mathrm{GeV}^{-2}$ & $\omega^{-1}=0.905 \mathrm{fm}$ & $r(\mathbf{f m})$ & $r \omega$ & $J_{0}=1$ & $\Delta J$ & $\Delta \boldsymbol{J}(\mathbf{c a l})$ \\
\hline$f^{\prime}(1515)$ & & & 0.7394 & 0.8167 & $J=2$ & 1 & 1.113 \\
\hline$\pi(135)$-Trajectory & $\alpha^{\prime}=0.8 \mathrm{GeV}^{-2}$ & $\omega^{-1}=0.1065 \mathrm{fm}$ & $r(\mathbf{f m})$ & $r \omega$ & $J_{0}=0$ & $\Delta J$ & $\Delta J($ cal $)$ \\
\hline
\end{tabular}

Table 2. Calculation of $\Delta J$ for the Four $\rho, \omega, a_{2}$ and $f$-Trajectories

\begin{tabular}{|c|c|c|c|c|c|c|c|}
\hline$\rho(770)$-Trajectory & $\alpha^{\prime}=0.880 G e V^{-2}$ & $\omega^{-1}=0.6682 \mathrm{fm}$ & $r(\mathbf{f m})$ & $r \omega$ & $J_{0}=1$ & $\Delta J$ & $\Delta J($ cal $)$ \\
\hline$\rho(1700)$ & & & 0.5958 & 0.8915 & $J=3$ & 2 & 1.994 \\
\hline$\omega(782)$-Trajectory & $a^{\prime}=0.923 G e \mathrm{~V}^{-2}$ & $\omega^{-1}=0.7118 \mathrm{fm}$ & $r(\mathbf{f m})$ & $r \omega$ & $J_{0}=1$ & $\Delta J$ & $\Delta J($ cal $)$ \\
\hline$\omega(1670)$ & & & 0.6289 & 0.8836 & $J=3$ & 2 & 1.982 \\
\hline$\alpha_{3}(2021)$ & & & 0.8445 & 0.7581 & $J=4$ & 2 & 1.94 \\
\hline$\alpha_{6}(2450)$ & & & 0.9604 & 0.8430 & $J=6$ & 4 & 3.688 \\
\hline$f_{2}(1270)$-Trajectory & $\alpha^{\prime}=0.801 \mathrm{GeV}^{-2}$ & $\omega^{-1}=1.003 \mathrm{fm}$ & $\mathbf{r}(\mathbf{f m})$ & $\mathbf{r} \omega$ & $\mathbf{J}_{0}=\mathbf{2}$ & $\Delta J$ & $\Delta J($ cal $)$ \\
\hline
\end{tabular}


Table 3. Calculation of $\Delta J$ for a Parabolic Relation

\begin{tabular}{|c|c|c|c|c|c|c|c|}
\hline $\boldsymbol{a}_{\mathbf{2}}(\mathbf{1 2 9 3})$-Trajectory & $\boldsymbol{\alpha}^{\prime}=\mathbf{0 . 5 9 8 7} \mathbf{G e V}^{-\mathbf{2}}$ & $\boldsymbol{\omega}^{-\mathbf{1}} \mathbf{\mathbf { 0 . 8 6 9 4 }} \mathbf{f m}$ & $\boldsymbol{r}(\mathbf{f m})$ & $\boldsymbol{r} \boldsymbol{\omega}$ & $\boldsymbol{J}_{\mathbf{0}}=\mathbf{2}$ & $\Delta \boldsymbol{J}$ & $\Delta \boldsymbol{J}(\mathbf{c a l})$ \\
\hline \hline$a_{6}(2441)$ & & & 0.7379 & 0.8694 & $J=6$ & 4 & 2.88 \\
\hline
\end{tabular}

All mesons trajectories, except for $\omega$, deviate from strict linearity, and the deviation should be more pronounced the heavier the meson. The relative errors in Table 2 are largest for the $a_{6}(2450)$ and $f_{6}(2510)$ resonances, 0.078, and 0.075, respectively. We might, therefore, try to replace the linear relation, (15), by a parabolic one [3]:

$\alpha_{\alpha}\left(m^{2}\right)=0.8759+0.5858 m^{2}+\frac{1}{2} \cdot 0.0681 m^{4}$

Table 3 shows that there is a marked deviation in the angular momentum difference, with a relative error of 0.28 , so that the linear, Regge, plot is far superior to a parabolic one. $\omega^{-1}$ was calculated using the intercept relation, (17), and not the slope, (16), for that would have given a value far too small.

Table 4 shows the angular momentum increments for baryons. Although all $\Lambda$ 's have been listed to lie on the same Regge trajectory [p. 147][4], it clearly violates the principle that a Regge trajectory produces a bound state or resonance every time it increases by a factor of two. Thus, the $\Lambda$ trajectory should be considered as a superposition of Regge

Table 4. Calculation of $\Delta J$ for Baryon-Trajectories

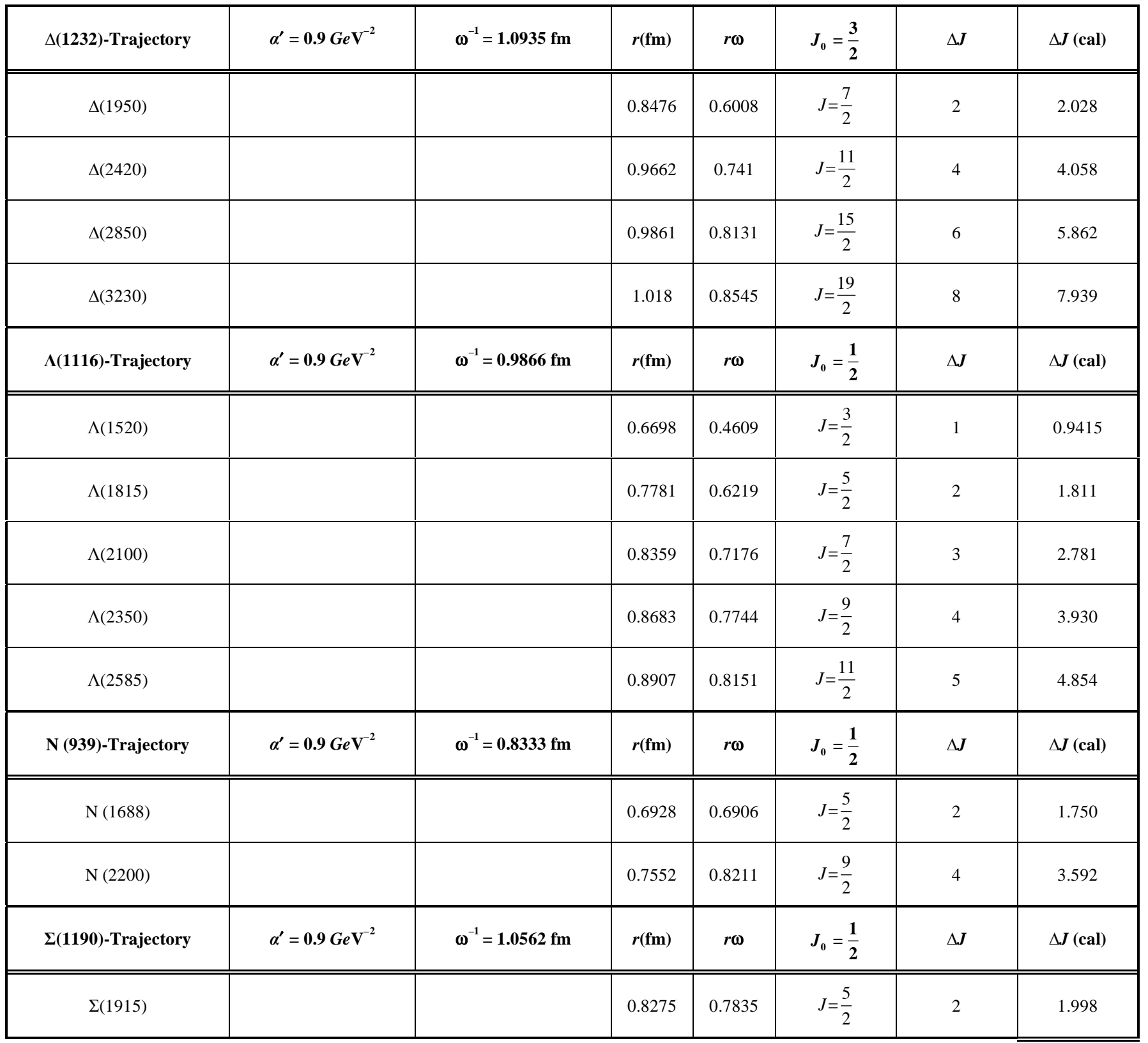


trajectories with different internal quantum numbers, and different signatures. Again, the relativistic expression, (11), for the angular momentum cannot discriminate among the trajectories with the same slope.

The intercept of the Regge trajectory dominates the scattering process, and from the optical theorem the asymptotic expression for the total cross-section is

$\sigma_{\text {tot }} \alpha s^{\alpha(0)-1}$.

Table 5 compares the observed and calculated values of the intercept, (17).

Table 5. Comparison of Observed and Calculated Values of the Intercept ${ }^{1}$

\begin{tabular}{|c|c|c|}
\hline Meson & $\boldsymbol{\alpha ( \mathbf { 0 } ) ( \mathbf { o b } )}$ & $\boldsymbol{\alpha ( \mathbf { 0 } ) ( \mathbf { c a l } )}$ \\
\hline \hline$\rho$ & 0.478 & 0.485 \\
\hline$\omega$ & 0.436 & 0.443 \\
\hline$a_{2}$ & 0.512 & 0.532 \\
\hline$f$ & 0.697 & 0.726 \\
\hline$K^{*}$ & 0.3 & 0.297 \\
\hline$\phi$ & 0.1 & 0.077 \\
\hline$\pi$ & 0 & -0.001 \\
\hline Baryon & $\boldsymbol{\alpha ( 0 )}(\mathbf{o b})$ & $\boldsymbol{\alpha ( 0 )}(\mathbf{c a l})$ \\
\hline \hline$N$ & -0.3 & -2.825 \\
\hline$\Delta$ & 0 & 0.153 \\
\hline$\Lambda$ & -0.6 & -0.60 \\
\hline$\Sigma$ & -0.8 & -0.757 \\
\hline
\end{tabular}

${ }^{1}$ The deviation between the observed and calculated values for $\Delta$ indicates that the intercept may not actually be zero as given in ref. [4].

\section{THE POMERON}

Chew and Frautschi [1] also drew attention to the existence of a Regge pole with $\alpha(0)=1$ that would carry the quantum numbers of the vacuum, and would be responsible for the forward scattering processes. Expression (20) indicates that as $\alpha(0) \rightarrow 1$, the total cross- section tends to a constant instead of vanishing asymptotically. It may, in fact, rise slowly with $s$ [10]. We will consider both cases. The critical trajectory has an intercept equal to 1 , while in the supercritical case it has an intercept greater than 1 . The exchange of a Reggeon, which cannot distinguish a particle from its antiparticle, is known as the Pomeron.

Expression (15) shows that for a slope, $\alpha_{p}^{\prime}=0.2$, the rest mass, $m_{0}=2.236 \mathrm{GeV}$. Such a particle would have a linear dimension of $\omega^{-1}=0.441 \mathrm{fm}$, and the various excited states are shown in Table 6, taking into account that the Pomeron has even signature.

The values in Table 6 may be compared with the supercritical Pomeron for which the total cross-section, (20), continues to rise as $\mathrm{s}$ increases. A slope of $\alpha_{p}=0.25 \mathrm{GeV}^{-2}$ gives a good fit to the data from elastic $p-p$ or $p-\bar{p}$ scattering over a range of $s$ at different $t$ values, and is the present generally accepted value [11]. The Regge trajectory will reach the value $\alpha_{p}(\mathrm{t})=2$ at a rest mass $\mathrm{m}_{0}=1.924 \mathrm{GeV}$, and intercept $\alpha_{p}(0)=1.075$. Values of $\Delta J$ are shown in Table 7.

Whereas the critical Pomeron trajectory has a rest mass of $16 \frac{1}{4}$ times the mass of the pion, the supercritical Pomeron trajectory will have a rest mass of $14 \frac{1}{2}$ times that of the pion. On the contrary, the size of the supercritical Pomeron has increased 1.07 times that of the critical Pomeron.

In conclusion, Regge trajectories, (15), are a consequence of the expression for the angular momentum change in hyperbolic space, (11). The identification of the corresponding term in relativistic conservation of energy,

$$
\begin{aligned}
\left(m_{0} c^{2}\right)^{2} & =E^{2}-(p c)^{2} \\
& =\left(m_{0} c^{2}\right)^{2}\left\{\cosh ^{2}(\bar{r} \omega / c)-\sinh 2(\bar{r} \omega / c)\right\} .
\end{aligned}
$$

with (11) gives the relativistic virial,

$$
2 \mathrm{KE}=p^{2} / m_{0}=\Delta J \omega \text {. }
$$

The change in the rotational energy is twice the kinetic energy.

\section{CONFLICT OF INTEREST}

The authors confirm that this article content has no conflict of interest.

Table 6. Calculation of $\Delta J$ for the Critical Pomeron-Trajectory

\begin{tabular}{|c|c|c|c|c|c|c|c|}
\hline $\mathbf{P}(\mathbf{2 2 3 6})$-Trajectory & $\boldsymbol{\alpha}^{\prime}=\mathbf{0 . 2} \mathbf{~ G e V}$ & $\boldsymbol{\omega}^{-\mathbf{1}} \mathbf{\mathbf { 0 . 4 4 1 }} \mathbf{f m}$ & $\boldsymbol{r}(\mathbf{f m})$ & $\boldsymbol{r} \boldsymbol{\omega}$ & $\boldsymbol{J}_{\mathbf{0}}=\mathbf{2}$ & $\Delta \boldsymbol{J}$ & $\Delta \boldsymbol{J}(\mathbf{c a l})$ \\
\hline \hline $\mathrm{P}(3873)$ & & & 0.3601 & 0.8165 & $J=4$ & 2 & 1.9772 \\
\hline $\mathrm{P}(5000)$ & & 0.3944 & 0.8944 & $J=6$ & 4 & 3.944 \\
\hline
\end{tabular}

Table 7. Calculation of $\Delta J$ for the Supercritical Pomeron-Trajectory

\begin{tabular}{|c|c|c|c|c|c|c|c|}
\hline $\boldsymbol{P}(\mathbf{1 9 2 4})-T r a j e c t o r y$ & $\boldsymbol{\alpha}^{\prime}=\mathbf{0 . 2 5} \mathbf{G e \mathbf { V } ^ { - \mathbf { 2 } }}$ & $\boldsymbol{\omega}^{-\mathbf{1}} \mathbf{\mathbf { 0 . 4 7 4 }} \mathbf{~ m}$ & $\boldsymbol{r}(\mathbf{f m})$ & $\boldsymbol{r \omega}$ & $\boldsymbol{J}_{\mathbf{0}}=\mathbf{2}$ & $\Delta \boldsymbol{J}$ & $\Delta \boldsymbol{J}(\mathbf{c a l})$ \\
\hline \hline $\mathrm{P}(3421)$ & & & 0.3922 & 0.8270 & $J=4$ & 2 & 1.974 \\
\hline $\mathrm{P}(4438)$ & & & 0.4274 & 0.9011 & $J=6$ & 4 & 3.942 \\
\hline
\end{tabular}




\section{ACKNOWLEDGEMENTS}

Declared none.

\section{REFERENCES}

Chew GFP, Frautschi SC. Regge trajectories and the principle of maximum strength for strong interactions. Phys Rev Lett 1962; 8: $41-4$.

[2] Johnson K, Thorn CB. Stringlike solutions of the bag model. Phys Rev D 1976; 13: 1934-9.

[3] Desgrolard P, Giffon M, Martynov E, Predazzi E. Exchangedegenerate Regge trajectories: a fresh look form resonance and forward scattering regions. Eur Phys J 2001; 18: 555-61.

[4] Collins PDB. An introduction to regge theory and high energy physics. Cambridge: Cambridge University Press 1977; p. 144.
[5] Frautschi SC, Ed. Regge poles and S-Matrix theory. New York: Benjamin WA 1963.

[6] O'Neill B Ed. Elementary differential geometry. New York: Academic Press 1966.

[7] Lavenda BH. A New Perspective on relativity: An Odyssey in Non-Euclidean Geometries. Singapore: World Scientific 2011; p. 437.

[8] Møller C. The theory of relativity. Oxford: Clarendon Press 1952; p. 241, Eq. (76a).

[9] Gottfried K, Weisskopf VF. Concepts of particle physics. vol. II New York: Oxford University Press 1986; p. 402.

[10] Forshaw JR, Ross DA. Quantum chromodynamics and the pomeron. Cambridge; Cambridge University Press 1997; p. 16.

[11] Frankfurt L, Strikman M. Diffractive phenomena in high energy processes. 2013 [Epub ahead of print].

(C) B.H. Lavenda; Licensee Bentham Open

This is an open access article licensed under the terms of the Creative Commons Attribution Non-Commercial License (http://creativecommons.org/licenses/by$\mathrm{nc} / 3.0 /$ ) which permits unrestricted, non-commercial use, distribution and reproduction in any medium, provided the work is properly cited. 